\title{
Études de communication : le dessous des cartes
}

A Citation Analysis of the Journal Études de communication

\section{Stéphane Chaudiron}

\section{(2) OpenEdition}

Journals

Édition électronique

URL : http://journals.openedition.org/edc/7706

DOI : 10.4000/edc.7706

ISSN : 2101-0366

Éditeur

Université de Lille

\section{Édition imprimée}

Date de publication : 1 juin 2018

Pagination : 191-222

ISBN : 978-2-917562-19-2

ISSN : $1270-6841$

Référence électronique

Stéphane Chaudiron, "Études de communication : le dessous des cartes », Études de communication

[En ligne], 50 | 2018, mis en ligne le 01 juin 2020, consulté le 05 janvier 2020. URL : http://

journals.openedition.org/edc/7706 ; DOI : 10.4000/edc.7706 
Études de communication : le dessous des cartes

A Citation Analysis of the Journal Études de communication 
Résumé / Abstract

À l'occasion de la parution du nu-

This article presents the results of méro 50 d'Études de communication, a bibliometric study of the French jourune étude bibliométrique de la revue a été réalisée dont l'objectif est l'ananal of information and communication sciences, Études de communication. In lyse de l'évolution des pratiques de citation au regard de la politique édiorder to study changes in citation practices over the last 25 years in relation toriale. L'analyse porte sur la période 1992-2016, qui correspond à la dénomination actuelle de la revue. L'article présente les premiers résultats de l'étude qui a été menée selon un triple point de vue : l'analyse des auteurs citants, l'analyse des auteurs cités et l'analyse des citations.

Mots-clés : Études de commuto the evolving editorial line of the journal, citation analysis was carried out on articles published during two periods, 1992-2007 and 2008-2016. We present the first results of this study, providing analysis of citing authors, cited documents and citation networks.

Keywords: Études de communication, bibliometrics, scientific journal, co-citation analysis, visualization. nication, analyse bibliométrique, pratiques de citation, co-citation, politique éditoriale, visualisation. 


\section{Introduction ${ }^{1}$}

Alors que, dans le domaine de la library and information science et de la communication science, les études portant sur la quantification de la littérature scientifique de langue anglaise sont nombreuses, peu de travaux existent sur la littérature francophone dans le domaine des sciences de l'information et de la communication. En library and information science (LIS), différentes études ont utilisé la méthode de l'analyse des co-citations pour visualiser le domaine, notamment White et McCain (1998), Åström (2007). Citons également les études de Schloegl et Stock (2004) qui ont étudié l'impact de 50 revues anglophones et allemandes en LIS, Bar-Ilan (2010) qui a établi le classement de 37 revues à partir de leur h-index et du facteur d'impact du Web of Science ou Hu et al. (2011) qui ont publié une analyse des co-citations de 24 revues LIS en Chine. En communication science (CS), de nombreux travaux s'appuient sur la présence des revues de langue anglaise dans le Journal Citation Reports - Social sciences pour identifier les thématiques centrales de recherche, l'influence des auteurs ainsi que les revues "dominantes » (Reeves et Borgman, 1983; Rice et al., 1988; Feeley, 2008; Chung et al., 2012).

En France, la revue Documentaliste - Sciences de l'information, fondée en 1976 par la fusion des revues Documentaliste (1964) et Sciences de l'information (1972) est probablement celle qui a intéressé le plus grand nombre de chercheurs. En 1997, Viviane Couzinet $(1997 a, 148)$ a dénombré six études consacrées à l'étude quantitative de son contenu, dont l'étude pionnière de Jeanine Cuypers en 1974 qui a comparé treize périodiques de bibliothéconomie et documentation en langue française. Viviane Couzinet s'est elle-même intéressée aux pratiques de citations des articles publiés dans la revue Documentaliste - Sciences de l'information (1997b) ainsi qu'aux «médiations hybrides » (2000) à l'œuvre dans le champ de l'infodoc, entre professionnels de la documentation et chercheurs. Soulignons également les travaux d'Hélène Prost et Joachim Schöpfel $(2012,64)$ qui ont étudié la place des revues du champ Information-Communication-Documentation (ICD) et «dressent un panorama de l'offre de périodiques francophones ICD en 2010, dans une double perspective scientifique et professionnelle ». Cette étude prolonge les réflexions initiées par Jeannin (2003), Rasse et al. (2010) et Heinderyckx (2012). Enfin, dans une récente publication (Chaudiron et Ihadjadene, 2017), nous avons tenté de montrer que la mise en place des procédures d'évaluation des laboratoires de recherche (ce que nous avons appelé «l'effet AERES») a modifié le paysage éditorial des revues scientifiques dans le sens d'une plus grande scientificité des procédures mais aussi, probablement, dans le sens d'une certaine normalisation. Pour démontrer cette hypothèse, nous

1 Cet article est une version corrigée de l'article initial. II a été mis en ligne le 19 décembre 2019. 
avons notamment utilisé une approche bibliométrique qui montre l'évolution des pratiques de citation pour le cas particulier de la revue Études de communication.

Néanmoins, il n'existe pas d'études systématiques sur les supports scientifiques de référence en SIC visant à cartographier la discipline afin d'identifier par exemple les réseaux des auteurs, les réseaux de citations ou d'identifier l'évolution des "fronts de recherche" comme cela est le cas pour les revues de langue anglaise (par exemple Åström, 2007) ou d'autres domaines (Dugué et al., 2016).

L'étude présentée dans cet article s'inscrit dans un projet initié en 2012 qui vise à analyser d'un point de vue quantitatif (bibliométrique) et qualitatif (analyse des «fronts de recherche») l'évolution de la revue Études de communication, d'un point de vue interne et externe. Pour ce numéro anniversaire, il s'agit de comprendre l'évolution de la revue d'un triple point de vue : l'analyse des auteurs citants, l'analyse des auteurs cités et l'analyse des citations. Ces analyses s'appuient notamment sur les résultats du travail de stage de master effectué par Émeline Cabre au sein de la revue Études de communication d'avril à juin 2017 (Cabre, 2017). Dans cet article, il s'agit de vérifier si les pratiques de citation des auteurs ont évolué, compte tenu de l'évolution même du projet éditorial de la revue. Nous fondant sur une approche diachronique quantitative, nous avons réalisé une analyse bibliométrique à partir des citations présentes dans les articles publiés entre 1992 et 2016, cette période correspondant à la dénomination actuelle de la revue. Deux grandes périodes sont identifiées, la période 1992-2007 qui correspond au projet éditorial initial d'une revue inscrite dans le champ des sciences de la communication et la période 2008-2016 qui correspond à l'ouverture de la revue vers les thématiques de l'information-documentation. De plus, ces deux périodes correspondent strictement aux deux grands moments du positionnement scientifique du laboratoire qui édite la revue.

\section{1. Méthodologie}

Études de communication ${ }^{2}$ est une revue disponible en version papier et électronique publiée par l'université de Lille avec le soutien du laboratoire GERiiCO. En 50 numéros, 35 ans d'existence (1982-2017) et trois dénominations successives, la revue est passée du statut de bulletin interne d'un centre de recherche au statut de revue internationale. Les évolutions successives de la revue sont en partie le reflet des mutations opérées par son éditeur scientifique puis son laboratoire d'appui, le CERTE (Centre de recherche en techniques d'expression), le CERTEIC (Centre de recherche en techniques d'expression, information et communication), GERiCO (Groupe de recherche interdisciplinaire en com- 


\begin{tabular}{|c|c|c|}
\hline Année & Numéro & Titre \\
\hline $1982-1986$ & 1 à 6 & Bulletin du CERTE \\
\hline $1986-1992$ & 7 à 12 & Bulletin du CERTEIC \\
\hline $1992-2006$ & 13 à 27 & Études de communication \\
\hline $2007-$ en cours & $28-$ en cours & Études de communication \\
\hline
\end{tabular}

Tableau 1: Évolution du nom de la revue

munication) et GERiiCO (Groupe d'études et de recherche interdisciplinaire en communication).

La période considérée pour l'étude bibliométrique est comprise entre 1992 et 2016 (soit les numéros 13 à 46) pour 24 ans de publication de la revue et 36 numéros (dont deux numéros spéciaux). Précisons que de 1992 à 2008, la revue a publié un numéro par an, à l'exception des années 1993, 1995, 1996, 2001 et 2007 où deux numéros ont paru; à partir de 2008, le rythme de parution est passé à deux numéros par an. Au sein de cette même période, la revue a connu deux sous-titres, également révélateurs d'une évolution, mais que nous ne considérons pas dans notre analyse : de 1992 ( $\left.n^{\circ} 13\right)$ à $2001\left(n^{\circ} 23\right)$, la revue était sous-titrée «Techniques d'expression, information, communication», témoin de l'ancien titre et de l'ancien laboratoire puis, à partir du numéro 24 (2001), la revue a porté comme sous-titre "Langages, information, médiations » jusqu'au numéro 44 (2015), date à laquelle celui-ci a disparu à l'occasion du renouvellement de la charte graphique de la revue. Sur l'ensemble de la période, un corpus de 309 articles a été recensé.

L'analyse prend en compte l'évolution éditoriale de la revue présentée dans (Chaudiron et Ihadjadene, 2017) qui distingue deux grandes périodes. À partir de 1992, sous sa nouvelle dénomination, Études de communication cherche à élargir sa visibilité au plan national avec un périmètre éditorial correspondant aux sciences de la communication. Pendant la période 1992-2007 (numéros 13 à 29), 151 articles sont publiés (hors notes de lecture). En 2007, la revue connaît une évolution significative de son projet éditorial avec l'élargissement de son périmètre scientifique à l'ensemble des sciences de l'information et de la communication (notamment l'information-documentation); 158 articles sont publiés au cours de la période 2008-2016 (numéros 30 à 46).

\subsection{Constitution du corpus}

Le corpus a été constitué en réalisant une extraction des références bibliographiques à partir du code source HTML des articles sur le site revues.org. $\mathrm{Ne}$ sont pris en compte que les articles des dossiers de recherche et des varias 
comprenant une bibliographie. Pour chaque article, le corpus comprend l'année de publication, le numéro de la revue, les pages de l'article, les auteurs citants, les auteurs cités, les références des citations et le titre de l'article citant.

Un problème de structuration des références bibliographiques a nécessité d'enlever le numéro 14 (année 1993) du corpus car, dans ce numéro intitulé Éducation, formation : le temps de l'industrialisation?, les références bibliographiques des articles du dossier ne sont pas mentionnées en fin de chaque article comme c'est l'usage mais regroupées dans le seul article Éléments de bibliographie ${ }^{3}$ de Viviane Glikman. Ce format ne permettant pas d'associer les auteurs citants aux auteurs cités, le numéro 14 n'a pas été pris en compte dans l'analyse bibliométrique. N'est pas non plus pris en compte le Supplément 1993 qui correspond aux actes d'un séminaire. En revanche, le numéro spécial de 2007 est intégré dans le corpus dans la mesure où il correspond aux mêmes critères de scientificité que les autres numéros. Le corpus final comprend donc 34 numéros publiés entre 1993 et 2016, c'est-à-dire 6060 références bibliographiques réparties dans 290 articles.

Un long travail d'homogénéisation du corpus a ensuite été nécessaire pour résoudre les problèmes des graphies divergentes dans les noms des auteurs, des différences d'ordre des noms des auteurs dans les cas de publications à auteurs multiples, de l'évolution des normes des références bibliographiques au fil des années (notamment en ce qui concerne les noms à particule). À titre d'exemple, le nom Michel de Certeau est structuré de cinq manières différentes comme le montre le tableau 1 (outre une coquille dans l'une des graphies du nom). Il a donc été nécessaire de normaliser les références bibliographiques.

\begin{tabular}{|c|l|l|}
\hline AuteursCitants & AuteursCites & ReferencesCitation \\
\hline Yannick Estienne & Certeau (de) M., 1990 & $\begin{array}{l}\text { (1990), L'invention du quotidien, tome 1: } \\
\text { Arts de faire, Paris, Gallimard, 347 p. }\end{array}$ \\
\hline Isabelle Comtet & Certeau de M., 1990 & $\begin{array}{l}\text { (1990), L'invention du quotidien, tome 1: } \\
\text { Arts de faire, Paris, Gallimard, 347 p. }\end{array}$ \\
\hline Yves Jeanneret & Carteau M. (de), 1975 & $\begin{array}{l}\text { (1975), L'Écriture de l'histoire, Gallimard, } \\
\text { Paris. }\end{array}$ \\
\hline Stefan Bratosin & Certeau M. de, 1990 & $\begin{array}{l}\text { (1990), L'invention du quotidien, tome 1: } \\
\text { Arts de faire, Paris, Gallimard, 347 p. }\end{array}$ \\
\hline Sylvette Giet & De Certeau M., 1975 & $\begin{array}{l}\text { (1975), L'Écriture de l'histoire, Gallimard, } \\
\text { Paris. }\end{array}$ \\
\hline
\end{tabular}

Tableau 2: Problème de structuration d'une référence bibliographique

Un autre problème a concerné la nécessité de normaliser les dates mentionnées dans les références bibliographiques. Afin de différencier les publications

3 Glikman V. (1993). "Éléments de bibliographie». In Études de communication, vol. 14, p. 151-157. https ://journals.openedition.org/edc/2743. 
d'un même auteur et d'une même année, l'usage est d'associer une lettre à l'année (1997a et 1997b par exemple). Or, d'un article à l'autre, la même mention de date ne renvoie pas nécessairement à la même publication citée, comme le montre le tableau 4. Dans cet exemple, les auteurs citants utilisent des mentions de date différentes (1997 et 1997a) pour une même publication.

\begin{tabular}{|c|r|l|l|}
\hline Numéro & AuteursCitants & AuteursCites & References Citation \\
\hline 27 & $\begin{array}{c}\text { Jacques Noyer, } \\
\text { Bernard Delforce }\end{array}$ & Charaudeau P., 1997 & $\begin{array}{l}\text { (1997), Le discours d'information } \\
\text { médiatique : la construction du miroir } \\
\text { social, Paris, Nathan. }\end{array}$ \\
\hline 22 & Jacky Simonin & Charaudeau P., 1997 & $\begin{array}{l}\text { (1997), Le discours d'information } \\
\text { médiatique : la construction du miroir } \\
\text { social, Paris, Nathan. }\end{array}$ \\
\hline 22 & Patrick Charaudeau & Charaudeau P., 1997a a & $\begin{array}{l}\text { (1997), Le discours d'information } \\
\text { médiatique : la construction du miroir } \\
\text { social, Paris, Nathan. }\end{array}$ \\
\hline 22 & Patrick Charaudeau & Charaudeau P., 1997b & $\begin{array}{l}\text { (1997), Grammaire du sens et de } \\
\text { l'expression, Hachette. }\end{array}$ \\
\hline
\end{tabular}

Tableau 3: Problème des dates dans les appels de références

\subsection{La visualisation des données}

La visualisation des données a été effectuée avec le logiciel libre Gephi ${ }^{4}$ dans l'environnement Windows. L'importation du corpus dans le logiciel s'est faite avec le module d'extension intitulé "Convert Excel and csv files to network" qui permet d'importer des fichiers au format Excel ou CSV dans Gephi, en les transformant directement en réseaux. Si le fichier importé contient des informations temporelles, le logiciel permet de créer des réseaux dynamiques. Avec l'import du corpus global, il est possible d'obtenir une visualisation des données par année, ou encore selon une période de plusieurs années.

La visualisation des données a été réalisée en utilisant l'algorithme de spatialisation Force Atlas 2 qui est une version améliorée de Force Atlas. II permet notamment de gérer des graphes de plus grande taille et simplifie le paramétrage de l'attraction/répulsion des nœuds du réseau. Les différents paramètres facilitent l'analyse et permettent de comprendre les spatialisations obtenues. Comme le soulignent les concepteurs de l'algorithme (Jacomy et al., 2012, 3), "we focused ForceAtlas2 on fluency and quality, because fluency is required by Gephi's manipulative user experience, and because researchers prefer quality over performance". 


\section{2. \\ Résultats et discussion}

Dans cette section, nous présentons les premiers résultats obtenus à l'issue de trois types d'analyse : l'analyse des auteurs citants, l'analyse des auteurs cités en nous interrogeant notamment sur le phénomène de l'auto-citation, et l'analyse des citations. Cette section reprend les résultats obtenus par É. Cabre (2017) dans le cadre de son stage de master effectué en avril-juin 2017 pour le compte de la revue Études de communication, dans le cadre d'un projet plus vaste d'analyse des pratiques de publication scientifique dont les premiers résultats ont été présentés dans (Chaudiron et Ihadjadene, 2017).

\subsection{L'analyse des auteurs citants}

La figure 1 présente une visualisation du réseau des auteurs citants pour l'ensemble de la période 1992-2016. La carte met en évidence la participation des auteurs à un ou plusieurs numéros de la revue. Chaque nœud représente un numéro auquel sont liés les auteurs des articles du numéro en question. La visualisation montre une forte dispersion des auteurs citants créant ainsi plusieurs sous-réseaux. Neuf numéros sont isolés, ce qui atteste que la très grande majorité des auteurs n'a contribué qu'à un seul numéro au cours de la période. Ce constat est renforcé par le fait que dans les cinq sous-réseaux identifiés, seuls 21 auteurs ont écrit plus d'un article.

Concernant la première période, on observe qu'un sous-réseau prédomine, reliant treize numéros par le biais d'auteurs qui, par ailleurs, sont ceux qui ont le plus publié par rapport au corpus global.

On peut remarquer que dans ce sous-réseau qui correspond à la première période, trois auteurs ont publié à la fois dans le numéro 23 et le numéro 26 (Thomas Lamarche, Stefan Bratosin et Bruno Raoul). Ces numéros traitent respectivement du thème de la communication dans les services aux publics et du développement des territoires en lien avec la communication. Le point fort de la thématique de la communication dans l'espace public et les territoires est ce qui relie la présence de ces auteurs au sein de ces numéros.

La visualisation correspondant à la seconde période est représentative de l'évolution des thématiques de la revue à partir du numéro 30 . À partir du n 31 , les numéros sont presque systématiquement écrits par de nouveaux auteurs et en terme de fréquence de publication, seuls cinq auteurs ont publié plus d'un article au cours de cette période, à savoir Olivier Le Deuff, Florence Thiault, David Douyère, et les paires Jacques Noyer / Bruno Raoul, et Cécile Tardy / Lise Renaud.

La comparaison des réseaux des auteurs citants entre les deux périodes de la revue montre la prédominance d'un certain nombre d'auteurs. L'analyse des 


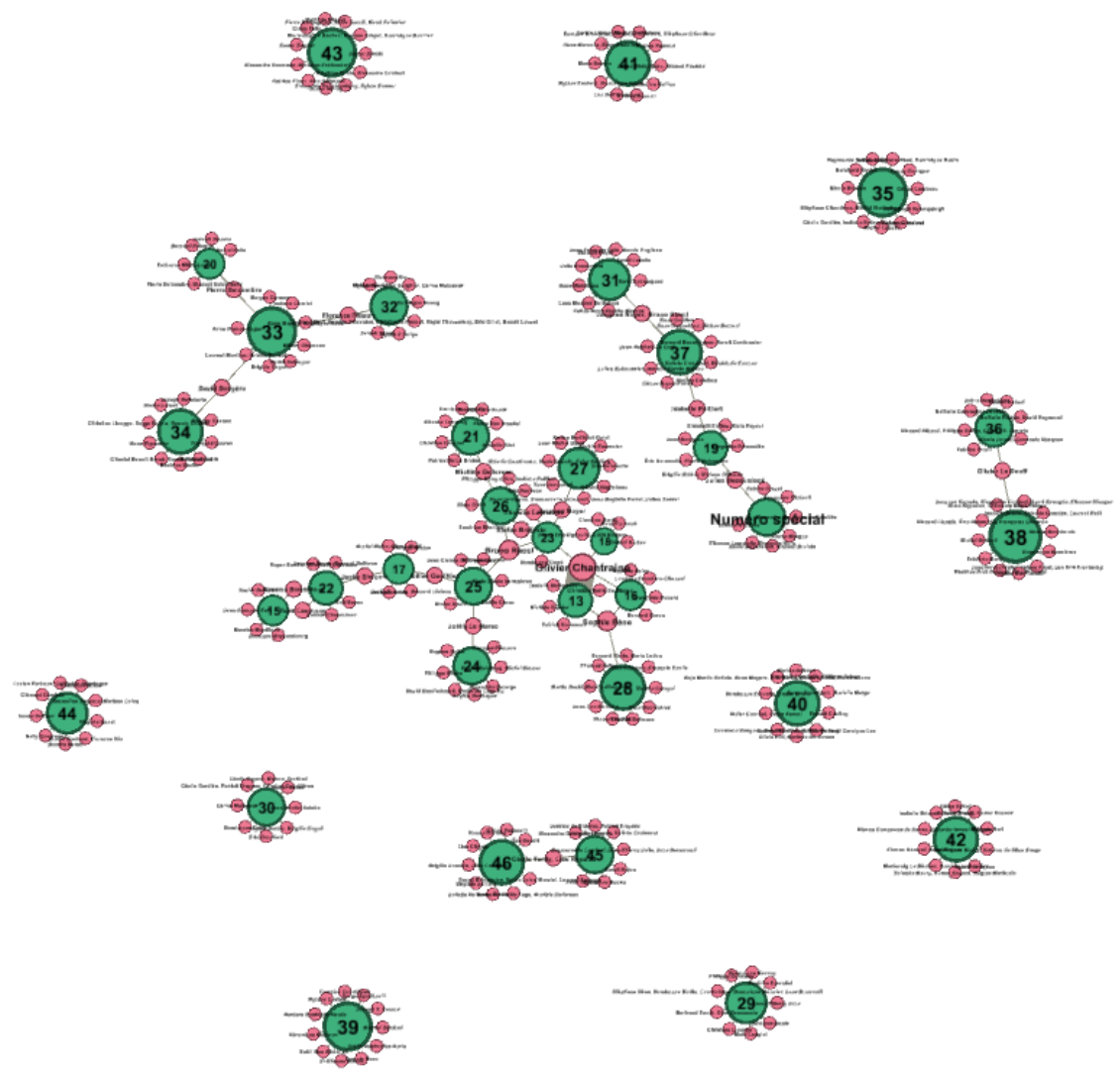

Figure 1: Visualisation des auteurs citants pour la période 1992-2016

21 auteurs ${ }^{5}$ ayant publié le plus d'articles au sein de la revue, en auteur unique ou en collaboration (cf. annexe 1), montre une forte représentation de membres du laboratoire GERiiCO. Les auteurs ayant le plus publié dans la revue (seuls ou en co-signature) sont Olivier Chantraine, Bruno Raoul et Jacques Noyer (à cinq reprises) et Pierre Delcambre (4 fois). On trouve ensuite 8 auteurs qui ont écrit à 3 reprises (Sophie Pène, Isabelle Pailliart, Jacky Simonin, Michèle Gellereau, Thomas Lamarche, Émilie Da Lage, Éric Delamotte et Anne Mayère). Le tableau de l'annexe 1 montre que c'est essentiellement dans la première période que ces auteurs ont le plus publié dans la revue, au moment où la ligne éditoriale de la revue ne s'est pas entièrement émancipée du laboratoire qui l'édite. Si on prend l'exemple de l'auteur qui a le plus publié d'articles au sein de la revue, à savoir

5 Le choix de ces 21 auteurs s'est fait par rapport à la fréquence de publication qui va de cinq à deux articles écrits. 


\begin{tabular}{|c|c|c|c|c|c|}
\hline Auteurs & $\nabla$ & Fréquence & Numéros - Période $1 \nabla$ & Numéros - Période $2 \mathbf{\nabla}$ & Université /Laboratoire \\
\hline Olivier Chantraine & & 5 & $13,16,18,23$ & & GERiCO - Lille 3 \\
\hline Bruno Raoul & & 5 (dt. 2) & $23,25,26$ & 31,37 & GERiiCO - Lille 3 \\
\hline Jacques Noyer & & 5 (dt. 3) & 23,27 & 31,37 & GERiiCO - Lille 3 \\
\hline Pierre Delcambre & & 4 (dt. 2) & 19,20 & 33 & GERICO - Lille 3 \\
\hline Michèle Gellereau & & 3 (dt. 1) & 21,26 & & GERiiCO - Lille 3 \\
\hline Thomas Lamarche & & 3 (dt. 1) & 23,26 & & GERiiCO - Lille 3 \\
\hline Émilie Da Lage-Py & & 3 (dt. 2) & 21,24 & 46 & GERiiCO - Lille 3 \\
\hline Éric Delamotte & & 3 (dt. 2) & 19,27 & 38 & Université de Rouen, puis GEriiCO - Lille 3 \\
\hline Florence Thiault & & 2 & & 32,33 & Rennes 2 (thèse à GERiiCO) \\
\hline Joëlle Le Marec & & 2 & 24,25 & & ENS Lyon (anciennement GERiiCO) \\
\hline Stefan Bratosin & & 2 & 23,26 & & Toulouse 3 (thèse à Lille 3 ) \\
\hline
\end{tabular}

Tableau 4: Fréquence de publication selon la période

Olivier Chantraine, le tableau montre que ce dernier a écrit cinq articles pour la revue au cours de la première période : deux articles dans le numéro 13 (1992), un article dans le numéro 16 (1995), un article dans le numéro 18 (1996) et un article dans le numéro 23 (2001).

Parmi ces vingt-et-un auteurs, onze ont été (ou sont) membres ou doctorants du laboratoire GERiCO ou GERiiCO selon la date, au moment de la publication de leurs articles : Olivier Chantraine, Bruno Raoul, Jacques Noyer, Pierre Delcambre, Michèle Gellereau, Thomas Lamarche, Émilie Da Lage-Py, Éric Delamotte, et Julien Deceuninck. Florence Thiault, Joëlle Le Marec, et Stefan Bratosin qui ont publié deux articles dans la revue dans la période 1992-2006, ont eu un lien avec le laboratoire à un moment donné de leur carrière. Le tableau 5 montre que la forte représentation de GERiiCO (ou GERiCO) parmi les auteurs concerne principalement la première période de la revue et est beaucoup moins sensible dans la seconde période. Cette évolution peut s'expliquer par le changement de projet éditorial, en particulier par la mise en place du système d'évaluation par comité de lecture en sélection en double aveugle qui participe de la volonté d'augmenter la scientificité de la revue ${ }^{6}$.

L'analyse de la visualisation en réseaux a montré que de nombreux auteurs récurrents ont publié plusieurs articles dans la première période, tandis que la deuxième période laisse davantage de place à de nouveaux chercheurs. En effet, dix des vingt-et-un auteurs étudiés ont publié uniquement dans la période 1992-2003. Parmi ces dix auteurs, sept viennent de laboratoires et universités différents, même si deux ont réalisé leur thèse à Lille 3 . Nous constatons que les quatre auteurs ayant oublié uniquement en deuxième période ne sont pas rattachés au laboratoire GERiiCO et proviennent des Universités de Toulouse 3, de Rennes 2, de Paris 13 et de Bordeaux 3. 
Cette première analyse confirme l'ouverture de la revue à partir de l'évolution de la ligne éditoriale de 2007 : outre l'ouverture thématique, elle est notablement moins endogène.

\subsection{Analyse des auteurs cités}

Comme nous l'avons indiqué, la revue a connu une progression du nombre d'auteurs à partir de la deuxième période, à savoir à partir de 2007. Concernant les auteurs cités, l'analyse en réseau confirme le caractère interdisciplinaire des sciences de l'information et de la communication, notamment dans le recours à des méthodes et/ou à des cadres théoriques issus de disciplines voisines, comme la sociologie, la psychologie sociale, la linguistique ou encore la philosophie. La proximité avec des chercheurs issus d'autres disciplines est observable dans l'analyse des auteurs les plus cités (cf. annexe 2). Parmi les trente auteurs les plus cités dans la revue, de nombreux chercheurs s'inscrivent dans le champ des sciences de l'information et de la communication, mais on observe également une forte présence de chercheurs issus de la sociologie, de la philosophie ou encore de l'anthropologie. Le réseau des auteurs cités confirme que c'est moins l'appartenance disciplinaire que le partage d'une certaine épistémologie de la recherche propre aux sciences sociales qui crée le réseau.

L'ouverture thématique de la revue à partir du numéro 30 marque, de nouveau, une rupture. Une nette augmentation des citations concernant des auteurs issus du domaine des sciences de l'information et de la communication est observable à partir 2007. Yves Jeanneret, Bernard Miège, François Cooren, Brigitte Guyot, Pierre Moeglin, et Jacques Perriault font partie de ces auteurs dont la fréquence de citation a crû en deuxième période. François Cooren est, avec le sociologue Alex Mucchielli, le seul auteur à n'être cité qu'à partir de 2007. Certains auteurs sont moins cités depuis l'évolution de la ligne éditoriale de la revue, comme Pierre Bourdieu, Jürgen Habermas, Eliséo Veron, ou encore Michel Callon. S'agit-il d'un effet de l'ouverture thématique de la revue ou d'un détachement actuel vis-à-vis des auteurs de référence? Cependant, tous les auteurs cités sur la période 1992-2000 le sont également dans la période suivante.

Le premier constat marquant dans l'analyse des œuvres les plus citées est la forte présence d'ouvrages. L'analyse des citations par type de documents que nous avons réalisée (Chaudiron et Ihadjadene, 2017) montre cette prédominance des ouvrages qui représente $48,16 \%$ des citations sur la période 1992-2016. De même, en ce qui concerne les quarante publications les plus citées dans la revue (annexe 2), le constat est encore plus marquant, puisque trente-deux sont des ouvrages, pour seulement quatre articles et quatre rapports. Ce résultat s'explique, selon Paul Rasse et al. $(2010,2)$, par la «place primordiale» des ouvrages dans les références en sciences humaines et sociales, tandis que les revues sont reléguées au second plan. En effet, les ouvrages sont encore dans ce domaine «le premier vecteur de la communication scientifique, assurent la 


\begin{tabular}{|c|c|c|c|c|}
\hline Auteurs & Titre & Fréquence - .t & Période 1.7 & Période 2 - \\
\hline Beuscart J.-S., Beauvisage T., Maillard S., 2012 & $\begin{array}{l}\text { La fin de la télévision ? Recomposition et } \\
\text { synchronisation des audiences de la télévision de } \\
\text { rattrapage, in Réseaux, } n^{\circ} 175, \text { pp. } 43-82 \text {. }\end{array}$ & 6 & - & is \\
\hline Cooren F., 2004 & $\begin{array}{l}\text { Textual Agency: How Texts Do Things in Organizational } \\
\text { Settings, in Organization, vol. 11, n' 3, pp. 373-393. }\end{array}$ & 6 & -. & 6 \\
\hline ERTe CN, 2005 & $\begin{array}{l}\text { Résultats } 2005 \text { de l'ERTe } \alpha \text { Modèles économiques et } \\
\text { enjeux organisationnels des campus numériques } *\end{array}$ & 6 & -. & 6 \\
\hline Missika J.-L., 2006 & La Fin de la télévision, Paris, Seuil, 108 p. & 5 & -. & 5 \\
\hline Combès Y., Moeglin P., 2005 & $\begin{array}{l}\text { « C@mpuSciences, d'un modèle industriel à l'autre », } \\
\text { accessible aux adresses indiquées à ERTe CN } 2005 .\end{array}$ & 5 & -- & 5 \\
\hline Fichez E., Benchenna A., 2005 & $\begin{array}{l}\text { Modèles économiques et enjeux organisationnels des } \\
\text { Campus numériques : le cas CampusCultura, accessible } \\
\text { aux adresses indiquées à ERTe CN } 2005 .\end{array}$ & 5 & - & 5 \\
\hline Akrich M., Callon M., Latour B., 2006 & $\begin{array}{l}\text { Sociologie de la traduction. Textes fondateurs, Presses } \\
\text { de l'École des Mines, Paris, } 303 \text { p. }\end{array}$ & 5 & - & 5 \\
\hline Guyot B., 2006 & $\begin{array}{l}\text { Dynamiques informationnelles dans les organisations, } \\
\text { Paris, Lavoisier. }\end{array}$ & 5 & - & 5 \\
\hline Jeanneret $Y ., 2008 a$ & $\begin{array}{l}\text { Penser la trivialité. Tome } 1 \text { : la vie triviale des êtres } \\
\text { culturels, Paris, Hermès. }\end{array}$ & 5 & -. & 5 \\
\hline Jenkins H., 2006 & $\begin{array}{l}\text { Convergence culture : where old and new media collide, } \\
\text { New-York, NY University Press. }\end{array}$ & 5 & -. & 5 \\
\hline Davallon J., 2006 & $\begin{array}{l}\text { Le don du patrimoine: une approche } \\
\text { communicationnelle de la patrimonialisation, Paris, } \\
\text { Hermès science - Lavoisier. }\end{array}$ & 5 & -- & 5 \\
\hline
\end{tabular}

Tableau 5: Fréquence de publication selon la période

publicité des connaissances mais aussi l'essentiel de la visibilité et de la notoriété des chercheurs ".

Toujours en se fondant sur les quarante publications les plus citées, nous observons une évolution entre la première et la seconde période. Selon Brown et Gardner (cité par Chtioui et Soulerot, 2006), la probabilité qu'un document soit cité augmente avec son âge. Cependant, nous constatons que cette hypothèse ne s'applique pas à la revue. En effet, les dates de publication des œuvres citées dans la période 2007-2016 sont majoritairement situées après les années 2000. De plus, dans la même période, se trouvent onze publications qui n'étaient pas présentes dans la première période, ceci étant dû à leur date de parution récente. Comme le montre le tableau ci-dessous (tableau 6), ces publications ont été publiées entre 2004 et 2012, pourtant leur fréquence de citation est déjà remarquable. Cette présence peut s'expliquer par la nature des thèmes traités, récents et qui correspondent aux thématiques des sujets traités dans la revue. Nous remarquons ainsi la forte présence de l'article La fin de la télévision? Recomposition et synchronisation des audiences de la télévision de rattrapage de J.-S. Beauscart et al., ainsi que l'ouvrage La fin de la télévision de J.-L. Missika dans la liste des publications les plus citées dans la seconde période. La citation de ces publications dans la seconde période est à mettre en lien avec le thème des pratiques télévisuelles à l'ère du numérique abordé pour la première fois dans la revue avec le numéro 44. L'arrivée des nouvelles thématiques notamment concernant le numérique donne lieu à l'apparition de citations concernant des productions plus récentes. Cette évolution, qui mériterait une analyse approfondie, semble confirmer une évolution plus générale de la revue qui privilégierait des thématiques portant sur des objets de recherche plus actuels. 
Inversement, nous constatons que certaines publications fortement citées durant la première période ont tendance à l'être moins dans la seconde, comme par exemple Surveiller et punir de Michel Foucault (de 9 à 3 citations) ou encore L'espace public de Jürgen Habermas (de 6 à 1 citation). Cette moindre fréquence concerne principalement les ouvrages les plus anciens, même si cela est à nuancer dans certains cas, puisque La mise en scène de la vie quotidienne d'Erving Goffman qui date de 1973 voit sa fréquence augmenter dans la seconde période. Ainsi, en élargissant ses thèmes de recherches à l'ensemble du champ des sciences de l'information et de la communication à partir de 2007, la revue a également connue une évolution des œuvres citées à partir de cette période qui conduit à l'émergence de nouvelles citations, plus récentes.

Nous terminerons cette analyse des citations en nous attardant sur les phénomènes de l'auto-citation et de l'auto-référence. Alors que l'auto-citation concerne les références bibliographiques qu'un auteur fait à propos de ses propres publications ou auxquelles il a collaboré, l'auto-référence désigne la citation d'articles publiés dans la revue Études de communication. Ces deux phénomènes sont qualifiés par Erwing et Garfield (cités par Chtioui et Soulerot, 2006) de "citations négatives ". Selon eux, certains auteurs auraient tendance à se citer ou citer des collègues au lieu de privilégier d'autres auteurs. Cependant, les travaux réalisés sur le sujet montrent que celles-ci auraient leur importance concernant la communication scientifique. Pour Hyland (cité par Chtioui et Soulerot, 2006), elles seraient « un moyen pour l'auteur de construire textuellement sa credibilite et sa trajectoire dans un domaine disciplinaire». Cette question mériterait une analyse approfondie mais quelques constats sont possibles dès à présent.

Le corpus global comprend 471 auto-citations, ce qui correspond à $7,77 \%$ du corpus (cf. annexe 3). L'analyse des auteurs qui se sont auto-cités cinq fois ou plus montre que ces auteurs sont majoritairement extérieurs au laboratoire GERiiCO (à part Olivier Chantraine et Pierre Delcambre). Olivier Chantraine est l'auteur se citant le plus mais ce chiffre est à nuancer dans la mesure où il a écrit cinq articles dans la revue, ce qui correspond à un total de 62 citations avec seulement 13 auto-citations, soit $21 \%$ du nombre de citations. Pour comprendre la pratique de l'auto-citation, il importe de considérer leur fréquence en fonction de l'activité de production scientifique mais aussi de "l'étroitesse » du champ de recherche de l'auteur. Prenant l'exemple de Hugues Hotier qui a écrit l'article De l'interprétation au cirque dans le numéro 24, on constate qu'il obtient un pourcentage d'auto-citations de $60 \%$. Cependant, en regardant ses références bibliographiques de plus près, nous constatons que ces références abordent toutes le thème du cirque éducatif, thème dont on peut penser qu'il est peu traité dans le domaine des SIC. Nous pouvons donc supposer que cet article est une partie ou une continuité de son travail de recherche, qu'il valorise grâce aux auto-citations. Autre exemple marquant, Georges Péninou, dans son article Des signes en publicité publié dans le numéro 24 également, n'a utilisé que des auto-citations comme références bibliographiques. 
Comme nous l'avons montré dans (Chaudiron et Ihadjadene, 2017), les revues les plus citées dans Études de communication sont Réseaux, Études de communication, Hermès et Sciences de la société. Le classement des auto-références selon leur fréquence (cf. annexe 4) montre que ce sont les articles de la période 1992-2006 qui sont le plus cités. Sur quinze auto-références, seulement trois sont de la période 2007-2016 : Espace de travail et logique documentaire de Dominique Cotte dans le numéro 30, La redocumentarisation, un défi pour les sciences de l'information de Jean-Michel Salaün toujours dans le numéro 30, et Se mouvoir au sein du monde de l'information : comment les personnes parlent de leur activité d'information de Brigitte Guyot dans le numéro 33. Nous remarquons pour ces articles s'inscrivent dans des thèmes relevant des sciences de l'information et de la documentation, abordés à partir de 2007. Les articles les plus cités de la première période concernent la question de la médiatisation $\left(n^{\circ} 22\right)$ et celle de l'écriture professionnelle ( $n^{\circ} 11$ et 29$)$. Cette deuxième analyse, encore partielle, témoigne à nouveau d'une forte évolution de la revue entre les deux périodes. Les publications citées dans la seconde sont globalement plus récentes, se rapprochant ainsi peut-être d'un modèle plus proche des sciences exactes. On peut en effet faire l'hypothèse que les thématiques des dossiers de la seconde période, consacrés notamment au document (30), au web (36), à l'organisation des connaissances $(39)$, au numérique $(45,46)$ citent plus volontiers des auteurs récents.

\subsection{Analyse des citations}

Concernant les pratiques de citation, la visualisation de l'ensemble du corpus constitue un réseau très vague puisqu'il comprend 5449 nœuds et 6019 liens (cf. annexe 5). Ce vaste réseau permet de faire plusieurs constats. Tout d'abord, nous pouvons rapidement repérer les auteurs citants qui citent le plus grâce à leur taille plus importante des nœuds, comme la paire Jacques Noyer / Bruno Raoul, Stéphane Héas et al., ou encore Andrea Resmini et Yves Jeanneret. Ces auteurs ne sont pas nécessairement ceux qui publient le plus dans la revue. Yves Jeanneret et Andrea Resmini n'ont par exemple publié qu'un article dans la revue.

L'algorithme Force Atlas 2 utilisé pour cette spatialisation repousse automatiquement les nœuds isolés vers l'extérieur du réseau, ce qui permet de remarquer que plusieurs nœuds d'auteurs sont sans lien commun avec d'autres auteurs. Parmi ces auteurs citants isolés, nous identifions deux numéros entiers : le numéro 13 de 1992 intitulé Pratiques d'écriture et champs professionnels et le numéro 25 de 2005, Questions de terrains. L'épaisseur du trait des liens entre les nœuds est proportionnelle à la fréquence des citations. On remarque ainsi dans la figure 2 , qu'Olivier Chantraine ou David Douyère ont tendance à citer plusieurs fois les mêmes publications dans leurs articles. Pour Olivier Chantraine qui a écrit cinq articles dans la revue, ces mêmes références sont faites dans les deux articles qu'il a publiés dans le numéro 13. La proximité thématique des deux articles, à savoir les écritures professionnelles, peut expliquer la similitude des références, 


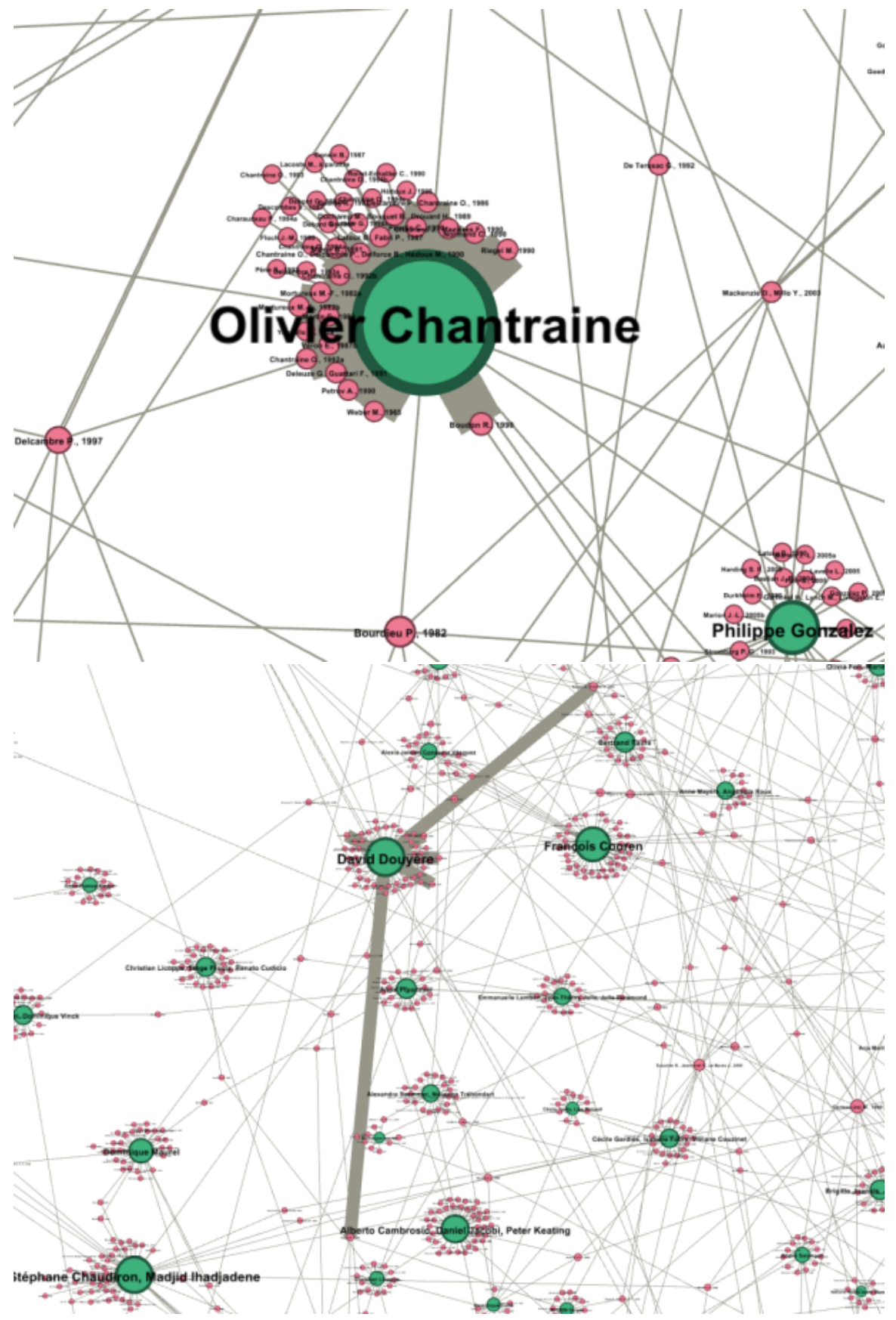

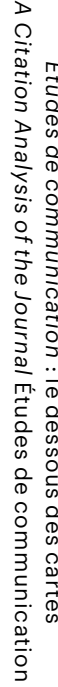

Figure 2: Extrait du réseau des citations (annexe 5)

d'autant plus qu'une grande part des recherches de l'auteur est centrée sur cette question. Quant à David Douyère, ce dernier a publié deux articles, un dans le numéro 33 et un dans le numéro 34, portant tous deux sur la thématique de la 
communication en entreprise, et nous pouvons également supposer que la même thématique explique l'utilisation d'un certain nombre de références identiques.

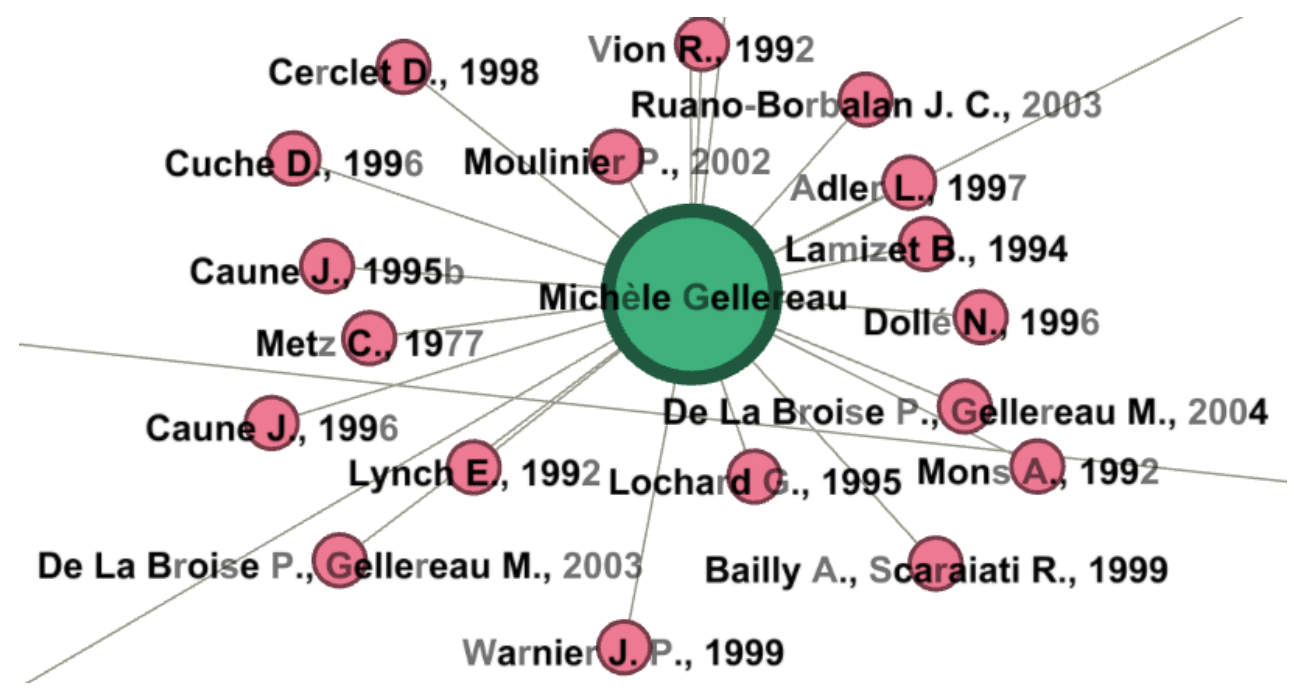

Figure 3: Extrait du réseau des citations (annexe 6)

Réaliser une comparaison des réseaux des deux périodes étudiées permet de se rendre compte d'une manière assez large de l'évolution de la pratique de citation. Les deux visualisations correspondant aux périodes 1992-2006 et 2007-2016 (cf. annexes 6 et 7) montrent une nette évolution. On observe tout d'abord l'augmentation du nombre d'auteurs citants, 116 dans la période 19922006 contre 174 dans la période 2007-2016. L'ouverture de GERiiCO à l'ensemble du champ des sciences de l'information et de la communication peut être une explication de cette augmentation, de même qu'une nouvelle ligne éditoriale et une périodicité régulière. L'augmentation du nombre d'auteurs citants coïncide avec l'augmentation du nombre de citations, comme le montre la densité accrue du réseau dans la deuxième période. La figure 3 donne un exemple du réseau de citations de Michèle Gellereau pour la première période.

L'analyse de l'évolution des citations par année de publication permet d'identifier les réseaux d'auteurs à partir de leurs citations communes. Plusieurs constats sont marquants. Un numéro entier ou quelques articles de celui-ci peut donner lieu à la constitution d'un réseau autonome. Par exemple, le numéro spécial de 2007 (figure 3) constitue un sous-réseau autonome dans lequel chaque auteur est relié par au moins un lien avec un autre. On peut ainsi supposer que la thématique du dossier de ce numéro, à savoir l'intégration du numérique dans les formations du supérieur, constitue un domaine de recherche extrêmement homogène, dense, qui relie entre eux à la fois les auteurs citants mais aussi les auteurs cités. Le même phénomène s'observe également dans les numéros 20 intitulé Communiquer... les mots de l'expérience, 28 Organisation, dispositif, 


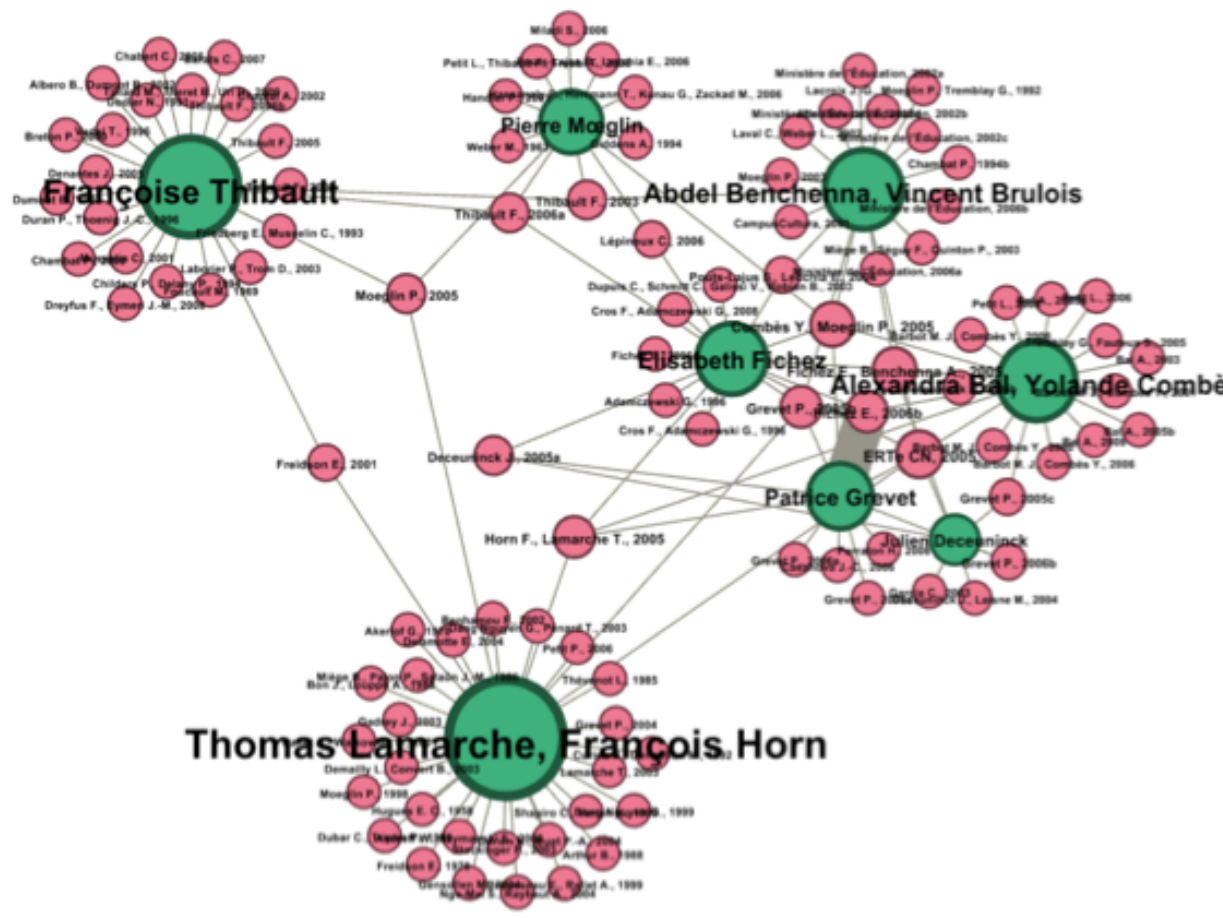

Figure 4: Carte du réseau des citations du numéro spécial de 2007

sujet, et 29 Performativité : relectures et usages d'une notion frontière. Cette forte concentration des citants-cités peut inversement être analysée comme un " entre-soi », signe d'une certaine fermeture (ou manque d'ouverture) à d'autres travaux, notamment non-francophones.

Il apparaît parfois que, grâce à des citations, un auteur citant constitue un lien vers d'autres auteurs. L'exemple d'Yves Jeanneret (figure 4) qui a écrit un article dans la revue en 2004 sur la monographie polyphonique et partage des citations avec quatre autres auteurs citants illustre cette situation. Par analogie avec la notion de «signe passeur » suggérée par (Davallon et Jeanneret, 2004, 46), nous pourrions qualifier ces auteurs d'auteurs passeurs.

De même, nous pouvons observer que la citation d'un ouvrage peut être source de lien entre différents auteurs d'un même numéro. C'est par exemple le cas de l'ouvrage Surveiller et Punir de Michel Foucault, qui a été cité cinq fois dans le numéro 28 Organisation, Dispositif, Sujet. II en est de même pour l'article de J.-S. Beuscart et al., intitulé La fin de la télévision? qui relie six auteurs dans le numéro 44 sur les pratiques télévisuelles à l'ère numérique (figure 6). Cette forte fréquence de ces auteurs dans ces numéros peut s'expliquer par la forte présence des publications et de leurs auteurs dans la thématique en question. 


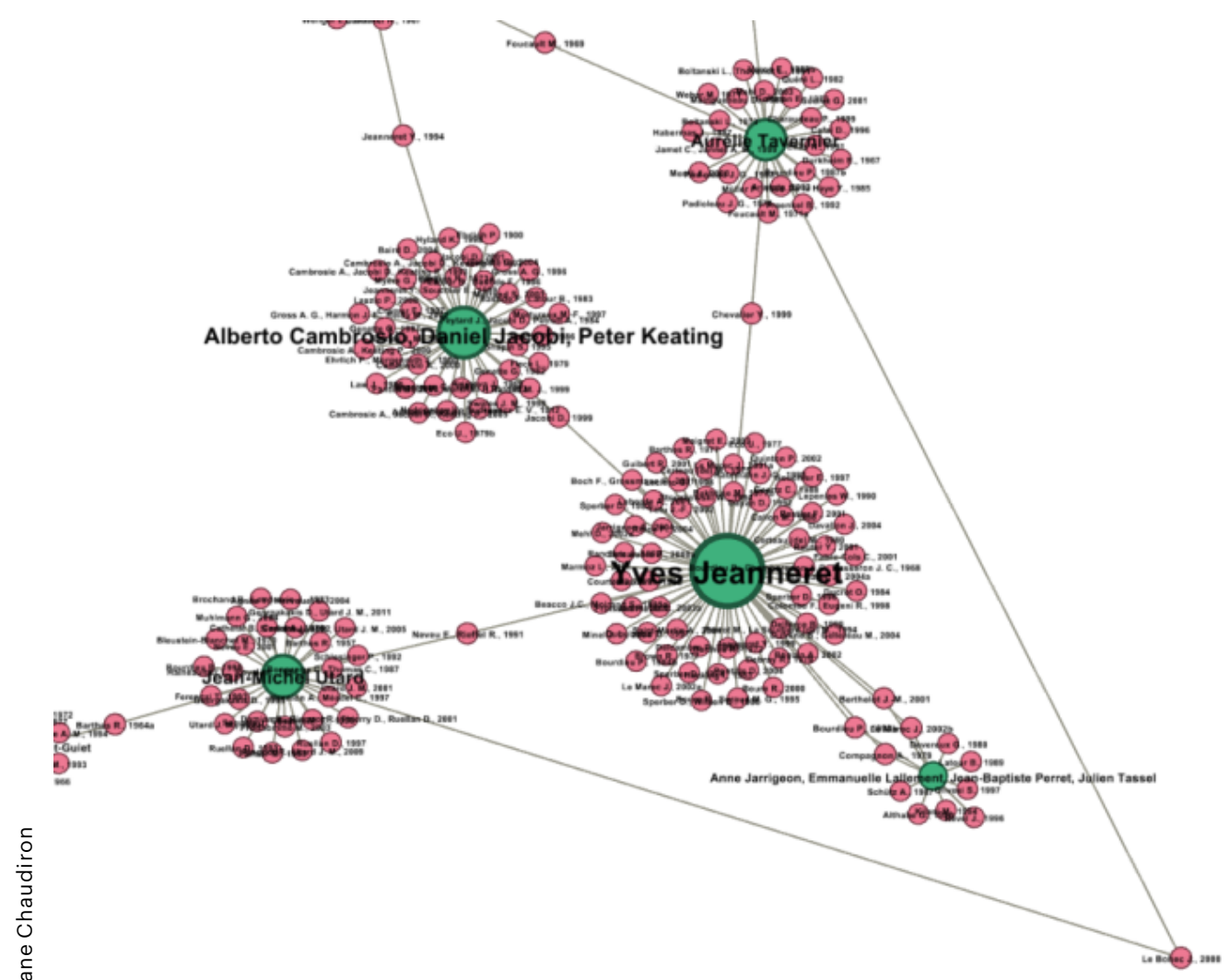

Figure 5: Exemple d'auteurs «passeurs»

\section{Conclusion}

Les quelques résultats présentés dans cette section sont loin d'épuiser l'analyse mais permettent de confirmer l'hypothèse selon laquelle les pratiques d'écriture et de citation ont fortement évolué à partir du changement de ligne éditoriale de la revue en 2007. Même partielle, l'analyse diachronique quantitative conforte ainsi l'analyse qualitative présentée dans (Chaudiron et lhadjadene, 2017). Ainsi, concernant les pratiques de signature des articles, nous avons observé que, "sur l'ensemble de la période, plus des deux tiers sont rédigés par un seul auteur (68,9\%) mais on constate que la pratique de co-écriture des articles a sensiblement augmenté les dix dernières années (période 2) ». De même, à propos des revues citées par les auteurs, nous notions qu'un autre résultat marquant est «l'apparition de trois revues de langue anglaise et clairement dans le champ des sciences de l'information (Library and information science) : Journal of the American Society for Information Science and Technology (Jasist), Journal of documentation et Organization Science » dans la seconde période. Cette 
Figure 6: Exemple de centralité d'une publication

évolution s'explique par l'ouverture de la revue Études de communication à des thématiques issues des sciences de l'information et du document.

Remerciements : Nous remercions Émeline Cabre pour sa participation à cette phase du projet et la qualité de ses analyses, ainsi qu'Abdou Beukeu Sow et Nicolas Hamez qui ont participé à cette étude dans le cadre de leur stage de master, respectivement en 2014 et 2015. 
Bibliographie

Åström F. (2007). "Changes in the LIS Research Front : Time-Sliced Cocitation Analyses of LIS Journal Articles, 1990-2004". In Journal of the American Society for Information Science, vol. 58, nº 7, p. 947-957.

Bar-llan J. (2010). “Rankings of information and library science journals by JIF and by h-type indices". In Journal of Informetrics, vol. 4, n ${ }^{\circ} 2$, p. 141-147.

Cabre E. (2017), Une analyse bibliométrique de la revue Études de communication, Mémoire de master 1 , Université de Lille 3. [Mémoire disponible sur https ://dumas.ccsd.c nrs.fr/dumas-01589750].

Chaudet B., Huet R., Tenier S., Malledant N., Thoa R., Sauvaire D. (2013). «La revue Études de communication ". In Revue française des sciences de l'information et de la communication, vol. 3, 2013.

Disponible sur http ://rfsic.revues.org/655 (page consultée le 2 octobre 2017)

Chaudiron S., Ihadjadene M. (2017). « Existe-t-il un 'effet AERES' sur les revues en SIC? Le cas d'Études de Communication ». In Fraysse P., Gardiès C. et Fabre I. (dir.), Sur les sciences de l'information et de la communication : contributions hybrides autour des travaux de Viviane Couzinet, Toulouse, Cépaduès éditions, p. 61-80.

Chtioui T., Soulerot M. (2006). "Quelle structure des connaissances dans la recherche en comptatbilité, contrôle et audit? Une étude bibliométrique de la revue $C C A$ sur la période 1995-2004 ». In Comptabilité Contrôle - Audit, vol. 12, n 1, p. 7-25.

Chung C. J., Barnett G., Kim K., Lackaff D. (2012). "An analysis on communication theory and discipline". In Scientometrics, vol. 95,

p. 985-1002.

Couzinet V. (1997a). « De l'information professionnelle à l'information scientifique : quelle place pour la recherche dans la revue Documentaliste-Sciences de l'information? ». In Documentaliste-Sciences de l'information, vol. 34, n³, p. 147-154.

Couzinet V. (1997b). «Pratiques professionnelles, pratiques de recherche : les articles de la revue Documentaliste-Sciences de l'information ». In Documentaliste-Sciences de l'information, vol. 34, n 6, p. 289-299.

Couzinet V. (2000). Médiations hybrides : le documentaliste et le chercheur en sciences de l'information, Paris, ADBS Éditions.

Couzinet V. (2008). «Évaluation de la recherche : cas des sciences de l'information et de la communication ". In Schedae, prépublication $\mathrm{n}^{\circ} 8$, fascicule 1, p. 77-86. Disponible sur https ://www.unicaen.fr/puc/images/ preprint0082008.pdf (page consultée le 2 octobre 2017)

Couzinet V. (2015a). Les revues : figures et cas, Toulouse, Cépaduès Éditions. 
Couzinet V. (2015b). «Ressources numériques dans l'espace européen ». In Les Cahiers du numérique, vol. 11, $\mathrm{n}^{\circ} 1$, p. 141-160.

Cuypers J. (1974). Étude comparative des périodiques de bibliothéconomie et de documentation en langue française, Mémoire de fin d'études : Bibliothécaires documentalistes, Bruxelles, Institut supérieur d'études sociales de l'État.

Davallon J., Jeanneret Y. (2004). «La fausse évidence du lien hypertexte». In Communication et langages, vol. 140, Dossier : Du «document numérique » au «textiel», p. 43-54. DOI : 10.3406/colan.2004.3266 (page consultée le 6 octobre 2017)

Delcambre P. (2008), «À partir de 1990, une autre étape de I'institutionnalisation des SIC ? ». In Actes du congrès de la SFSIC 2008. Disponible sur https : //www.sfsic.org/congres_2008/spip. php ?page=imprime\&id_article $=59$ (page consultée le 2 octobre 2017)

Dugué N., Lamirel J.-C., Cuxac P. (2016). «Visualisation pour la détection d'évolutions dans des corpus de publications scientifiques. Indexation, classification et analyse diachronique pour la visualisation $»$. In Les Cahiers du numérique, vol. $12, n^{\circ} 4$, p. 157-184.

Feeley T. (2008). "A bibliometric analysis of communication journals from 2002 to 2005". In Human Communication Research, vol. 34, $n^{\circ} 3$, p. 505-520.

Heinderyckx F., Hardy M., Vanholsbeeck M. (2012). «Les revues scientifiques en information- communication. L'ère des mutations? ". In Questions de communication, vol. 21, p. 157-170. Hu C.-P. et al. (2011). "A journal co-citation analysis of library and information science in China". In Scientometrics, vol. 86, $\mathrm{n}^{\circ} 3$, p. 657-670. Disponible sur http ://www.akademiai.com/content/ m7n221w83h61mt55/fulltext.pdf (page consultée le 4 octobre 2017).

Jacomy M., Heymann S., Venturini T., Bastian M. (2012), "ForceAtlas2, A Continuous Graph Layout Algorithm for Handy Network Visualization". Disponible sur https ://medialab.scien cespo.fr/publications/Jacomy_Heyma nn_Venturini-Force_Atlas2.pdf (page consultée le 5 juin 2019).

Jeannin P. (2003). Revuemétrie de la recherche en sciences humaines et sociales. Rapport synthétique et final de mission (1999-2003), Direction de la recherche, Ministère délégué à la recherche et aux nouvelles technologies. Disponible sur http ://www.revues.org/cost/images/1 /12/JEANNIN_-_Rapport_final2003a.pdf (page consultée le 4 octobre 2017).

Mouchon J. (1992). «Voici donc Études de Communication ». In Études de communication, vol. 13, p. 9.

Disponible sur http ://edc.revues.org/2751 (page consultée le 2 octobre 2017).

Prost H., Schöpfel J. (2012). Les périodiques francophones dans le domaine information, communication et documentation : une étude empirique. In Documentaliste Sciences de l'Information, vol. 49, n 3 , p. 62-70. 
Rasse P., Pélissier N., Colin M., Fleuret É., Gresillaud S., Guichard M., Hoel P. et Rageot L. (2010). "Panorama du paysage des revues de sciences de l'information et de la communication avant qu'il ne change ». In Communication, vol. $27, \mathrm{n}^{\circ} 2$, p. 217-233.

Reeves B., Borgman C. (1983). "A bibliometric evaluation of core journals in communication research". In Human Communication Research, vol. 10, n¹, p. 119-136.

Rice R., Borgman C., Reeves B. (1988). "Citation networks of communication journals, 1977-1985 : Cliques and positions, citations made and citations received". In Human Communication

Research, vol. 15, n² 2, p. 256-283.

Schloegl C., Stock W. G. (2004), "Impact and Relevance of LIS Journals : A Scientometric Analysis of International and German-Language LIS Journals - Citation Analysis Versus Reader Survey". In Journal of the American Society for Information Science and Technology, vol. 55, $n^{\circ} 13$, p. 1155-1168.

White H., McCain K. (1998).

"Visualizing a discipline : An author co-citation analysis of information science, 1972-1995". In Journal of the American Society for Information Science, vol. 49, $n^{\circ}$ 4, p. 327-355. 
Figure 1: Auteurs qui publient le plus fréquemment dans la revue

\begin{tabular}{|c|c|c|c|c|c|c|c|c|c|c|c|c|c|c|c|c|c|c|c|}
\hline 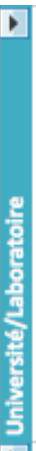 & 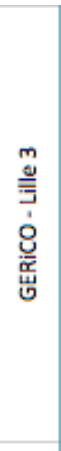 & 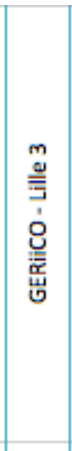 & 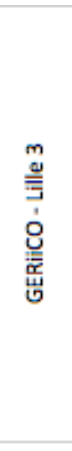 & 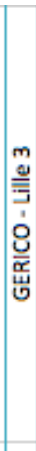 & 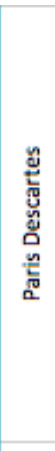 & 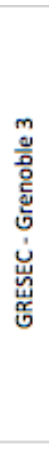 & 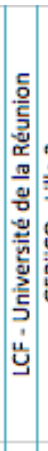 & 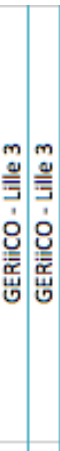 & 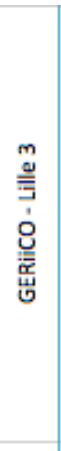 & 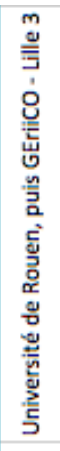 & 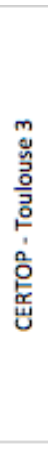 & 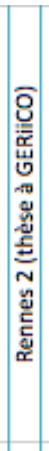 & 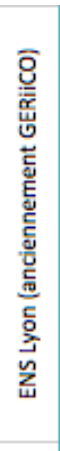 & 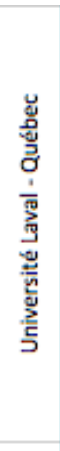 & 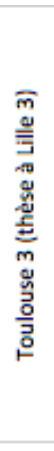 & 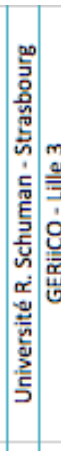 & 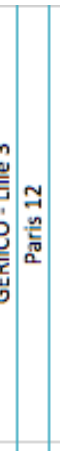 & 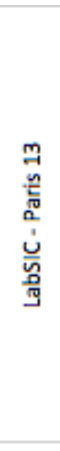 & 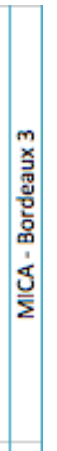 \\
\hline प्ष & & $\begin{array}{l}\hat{m} \\
\text { नें }\end{array}$ & $\begin{array}{l}\hat{m} \\
\text { ने }\end{array}$ & m & & $\hat{m}$ & & & 电 & $\stackrel{\infty}{m}$ & $\begin{array}{l}\text { \& } \\
\text { gे } \\
\text { ले }\end{array}$ & $\begin{array}{c}m \\
m \\
\tilde{m} \\
\text { m }\end{array}$ & & & & 证 & & $\begin{array}{l}\text { m } \\
\text { mे }\end{array}$ & $\left|\begin{array}{c}\infty \\
m \\
心 \\
心 \\
m\end{array}\right|$ \\
\hline 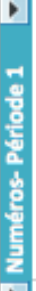 & 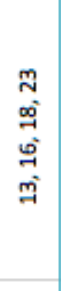 & 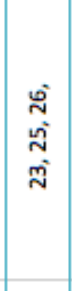 & $\begin{array}{l}\tilde{N} \\
\dot{N}\end{array}$ & $\begin{array}{l}\text { ని } \\
\text { gి }\end{array}$ & 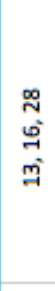 & $\begin{array}{l}2 \\
\text { g } \\
\text { ᄀ. }\end{array}$ & $\begin{array}{l}\tilde{N} \\
\tilde{\approx}\end{array}$ & & $\begin{array}{l} \pm \\
\text { N }\end{array}$ & $\begin{array}{l}\text { N } \\
\text { gે }\end{array}$ & & & $\begin{array}{l}\text { N } \\
\text { N }\end{array}$ & $\begin{array}{l}\text { N } \\
\stackrel{-1}{-1}\end{array}$ & $\begin{array}{l}\stackrel{2}{2} \\
\text { mi }\end{array}$ & 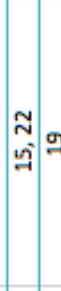 & & & \\
\hline 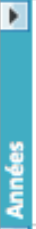 & 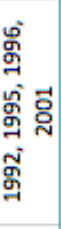 & 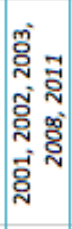 & 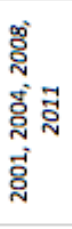 & 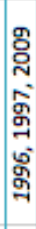 & 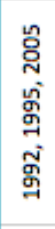 & 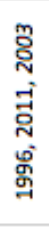 & 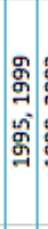 & 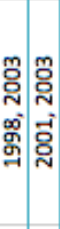 & 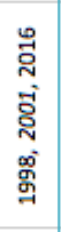 & 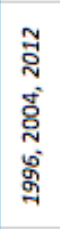 & 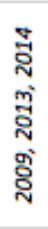 & ठ্ণ & 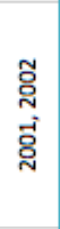 & 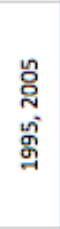 & 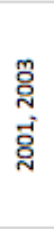 & 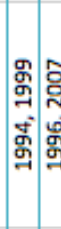 & 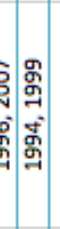 & 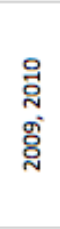 & 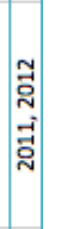 \\
\hline 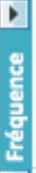 & in & $\begin{array}{l}\bar{N} \\
\text { in } \\
\text { in }\end{array}$ & $\begin{array}{l}\bar{m} \\
\text { tu } \\
\text { in }\end{array}$ & $\begin{array}{l}\bar{N} \\
\text { गे } \\
\frac{v}{4}\end{array}$ & m & $\begin{array}{l}\overline{7} \\
\text { 苛 } \\
\text {. }\end{array}$ & $\begin{array}{l}\bar{a} \\
\overrightarrow{0} \\
\mathrm{~m}\end{array}$ & $\begin{array}{l}\vec{z} \\
\text { ज्ञ } \\
\text { m }\end{array}$ & 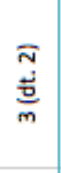 & $\begin{array}{l}\bar{N} \\
\text { Dे } \\
\text { D }\end{array}$ & $\begin{array}{l}\bar{m} \\
\text { 苛 } \\
\text { non }\end{array}$ & $N$ & $N$ & $N$ & $N$ & & $N \sim$ & $N$ & $N$ \\
\hline 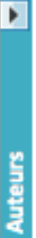 & 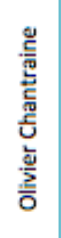 & 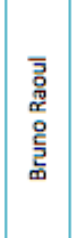 & 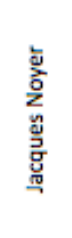 & 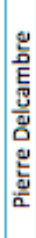 & 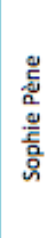 & 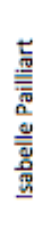 & 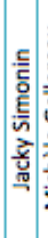 & 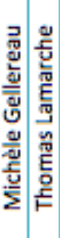 & 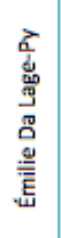 & 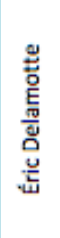 & 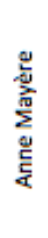 & 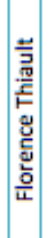 & 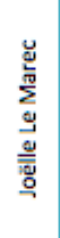 & 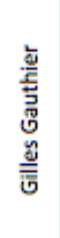 & 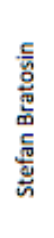 & 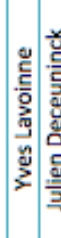 & 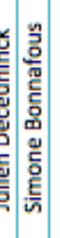 & 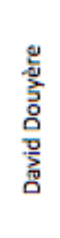 & 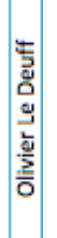 \\
\hline
\end{tabular}


Figure 2: Publications les plus citées

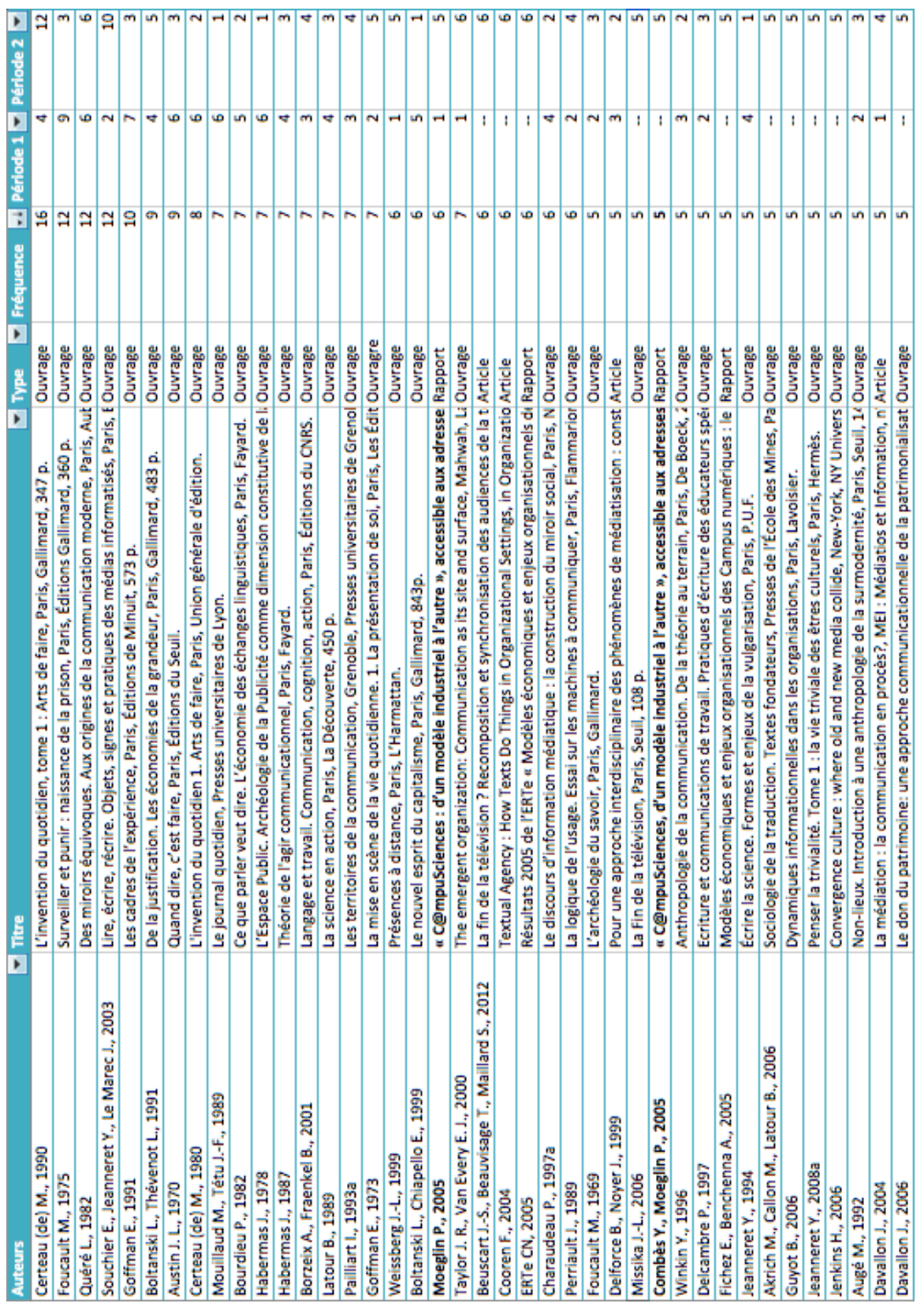


Figure 3: Analyse des auto-citations

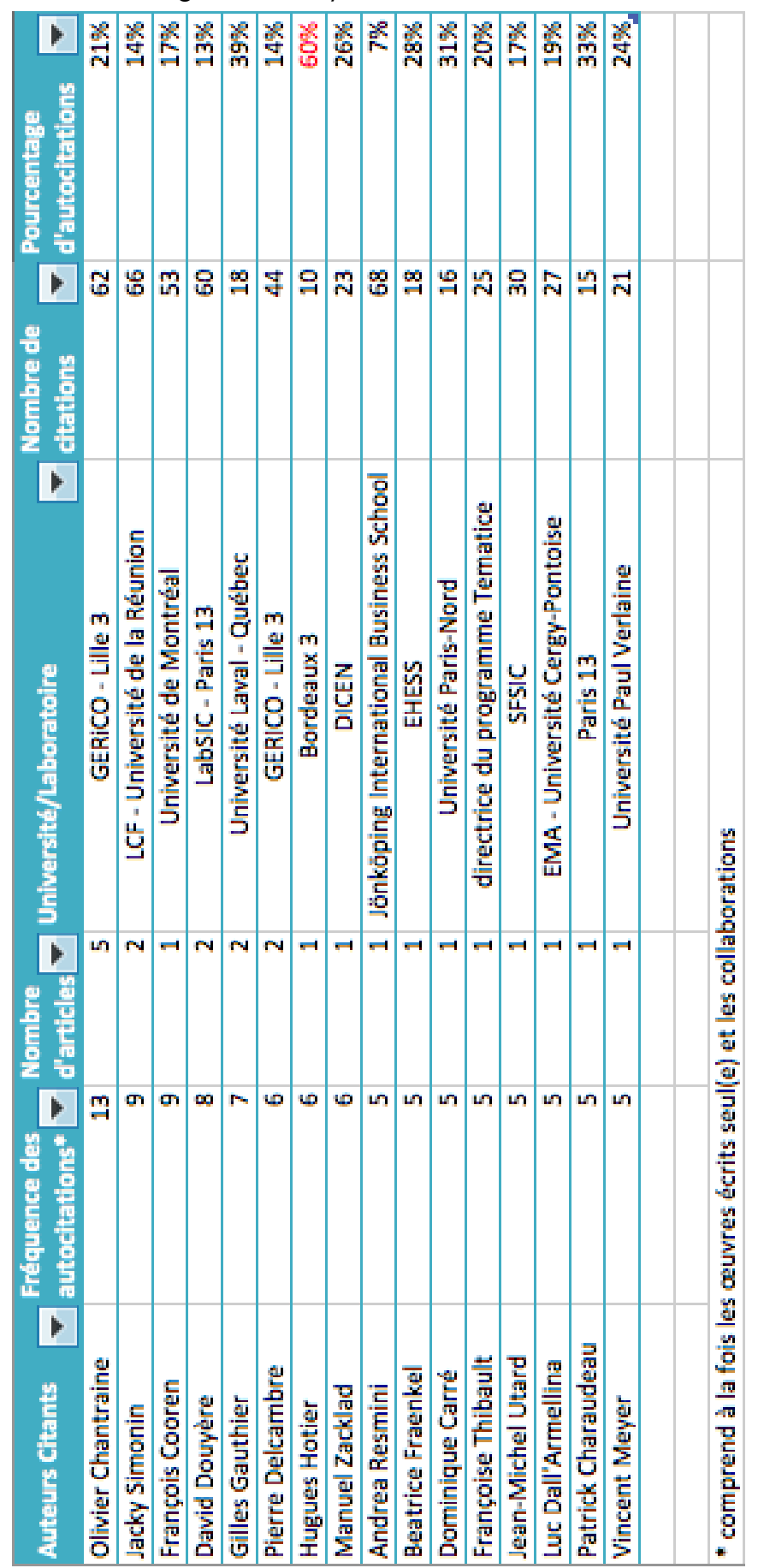

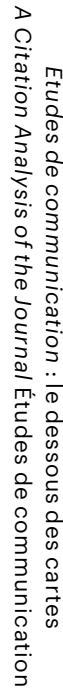


Figure 4: Analyse des auto-références

\begin{tabular}{|c|c|c|c|c|}
\hline Auteurs & Fréquence & $\mathbf{\nabla}$ & Titre de l'article & Numéro \\
\hline Delforce B., Noyer J., 1999 & & 5 & $\begin{array}{l}\text { Pour une approche interdisciplinaire des phénomènes de } \\
\text { médiatisation : constructivisme et discursivité sociale }\end{array}$ & 22 (1999) \\
\hline Delcambre P., 1990a & & 3 & $\begin{array}{l}\text { Écritures professionnelles et pouvoirs : Analyse d'un dispositif } \\
\text { d'écriture }\end{array}$ & $11(1990)$ \\
\hline Cotte D., 2007b & & 3 & Espace de travail et logique documentaire & $30(2007)$ \\
\hline Fraenkel B., 2006b & & 3 & $\begin{array}{l}\text { Actes écrits, actes oraux, la performativité à l'épreuve de } \\
\text { l'écriture }\end{array}$ & $29(2006)$ \\
\hline Caune J., 1993 & & 2 & De l'influence de la communication sur la diffusion artistique & $12(1991)$ \\
\hline Charaudeau P., 1999 & & 2 & $\begin{array}{l}\text { La médiatisation de l'espace public comme phénomène de } \\
\text { fragmentation }\end{array}$ & $22(1999)$ \\
\hline Delcambre P., 1990b & & 2 & $\begin{array}{l}\text { Une analyse d'écriture revisitée : ou, quand l'analyse d'écriture } \\
\text { fait intervention, que dire de ses effets? }\end{array}$ & $11(1990)$ \\
\hline Le Marec J., 2002b & & 2 & Questions de terrains & $25(2002)$ \\
\hline Guyot B., 2009 & & 2 & $\begin{array}{l}\text { Se mouvoir au sein du monde de l'information : comment les } \\
\text { personnes parlent de leur activité d'information }\end{array}$ & $33(2009)$ \\
\hline Bratosin S., 2003 & & 2 & $\begin{array}{l}\text { Grands Projets de Ville : un lieu de production symbolique du } \\
\text { territoire }\end{array}$ & $26(2003)$ \\
\hline Chantraine 0., 1992a & & 2 & $\begin{array}{l}\text { Les écritures professionnelles ou la difficile synthèse de } \\
\text { normes communicationnelles hétérogènes }\end{array}$ & $13(1992)$ \\
\hline Chantraine O., 1992b & & 2 & $\begin{array}{l}\text { Quelques exemples pour l'identification des espaces socio- } \\
\text { discursifs dans les écritures professionnelles }\end{array}$ & $13(1992)$ \\
\hline Raoul B., 2003 & & 2 & $\begin{array}{l}\text { Le développement des territoires au miroir de la } \\
\text { communication : une problématique en perspective }\end{array}$ & $26(2003)$ \\
\hline Salaûn J.-M., 2007 & & 2 & $\begin{array}{l}\text { La redocumentarisation, un défi pour les sciences de } \\
\text { l'information }\end{array}$ & $30(2007)$ \\
\hline $\begin{array}{l}\text { Chantraine O., Delcambre P., Delforce } \\
\text { B., Hédoux M., } 1990\end{array}$ & & 2 & Pratiques d'écriture et champs professionnels & $11(1990)$ \\
\hline
\end{tabular}


Figure 5: Réseau des citations sur l'ensemble du corpus

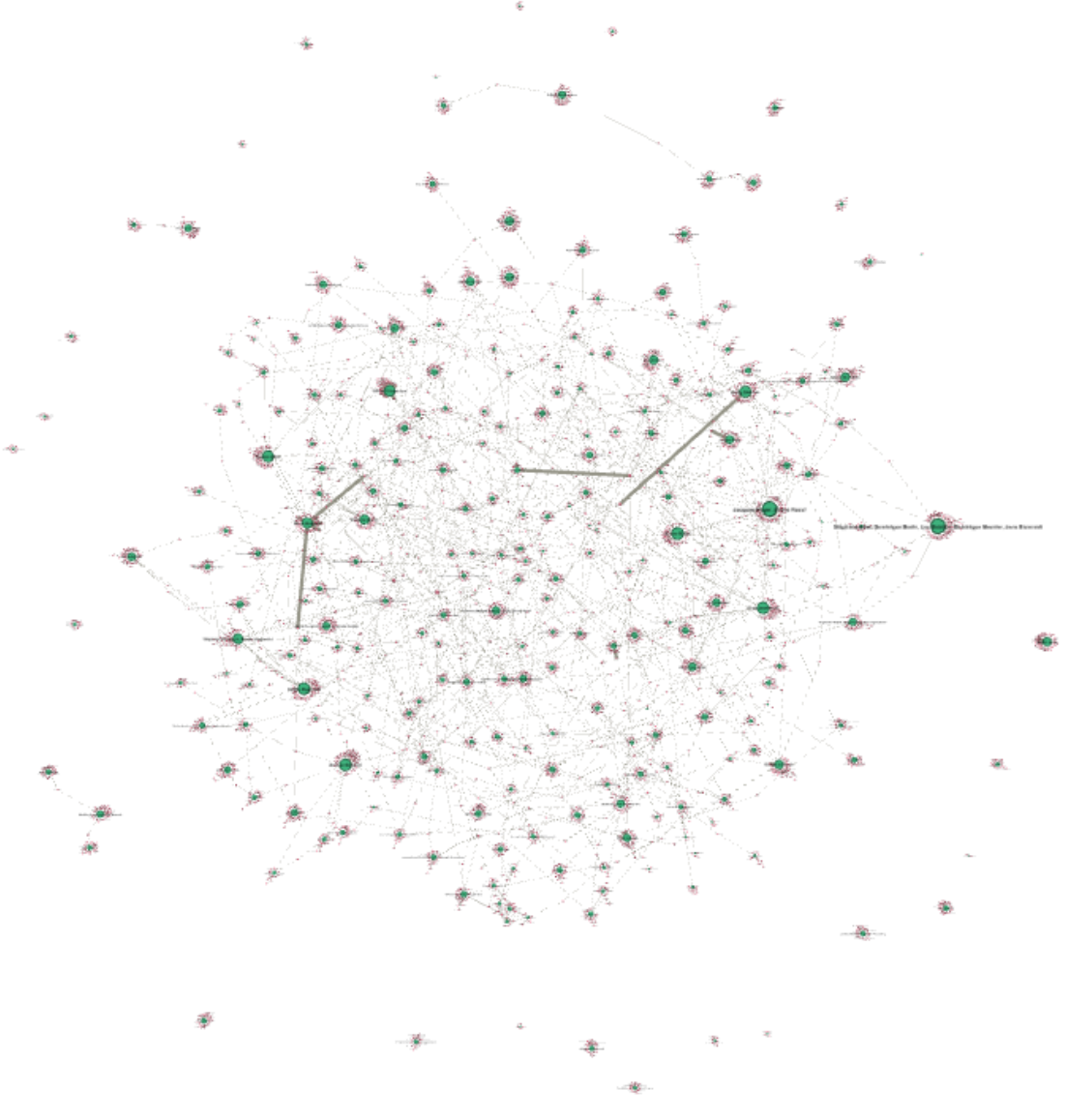


Figure 6: Visualisation du réseau des citations de la période 1992-2006 
Figure 7: Visualisation du réseau des citations de la période 2007-2016

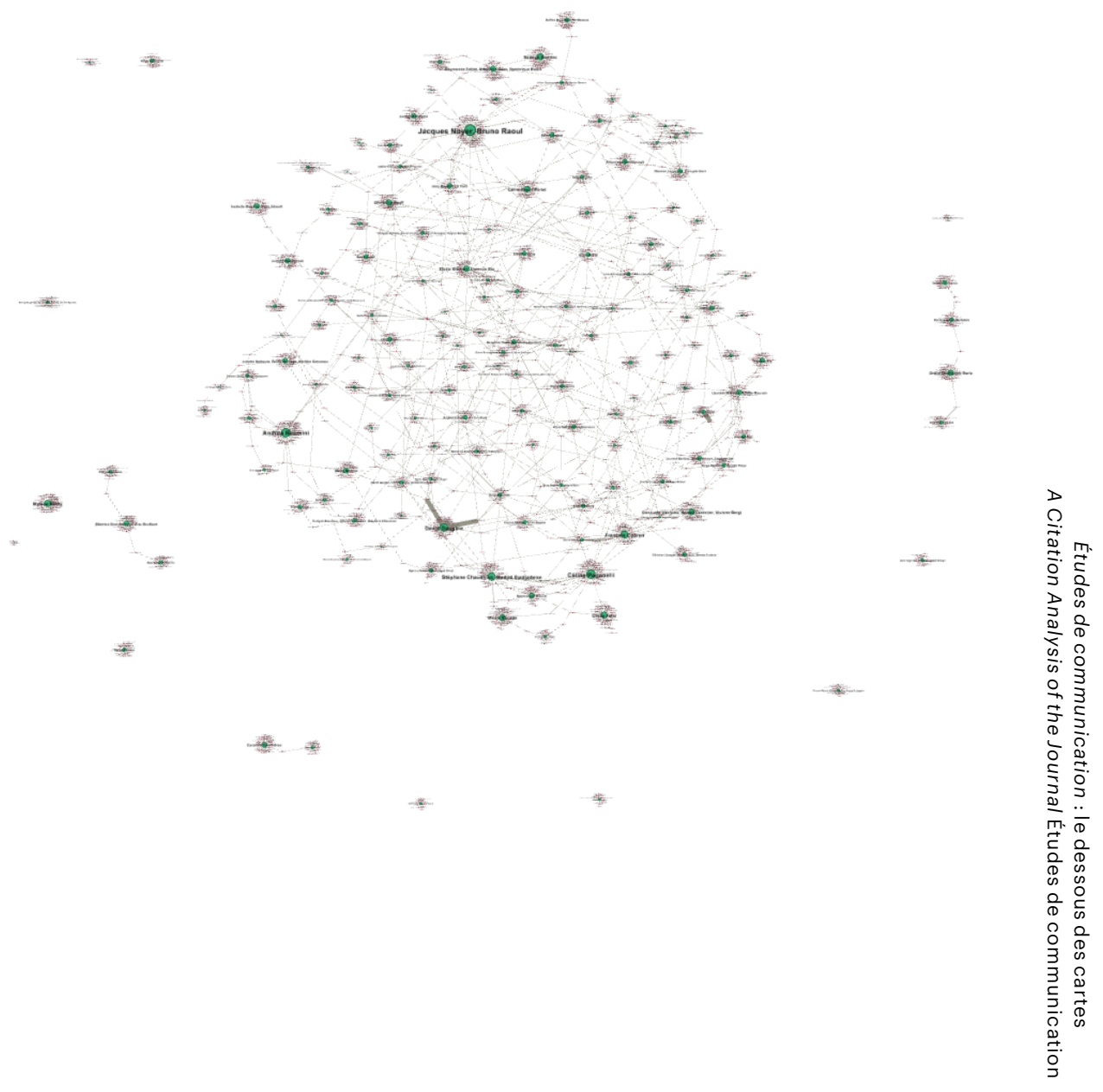

